\title{
HIGHLIGHTS
}

ATRIAL FIBRILLATION

\section{Strict heart-rate control is not necessary in patients with $\mathrm{AF}$}

Van Gelder and colleagues have reported that a lenient rate-control strategy is associated with outcomes comparable to those of strict rate control in patients with permanent atrial fibrillation (AF). Rate control is a widely used therapy for persistent AF, particularly among patients older than 65 years. However, a target heart rate has not been determined and there is concern that strict rate control, with increased doses of drugs, could be associated with adverse effects.

The RACE II study was a prospective, randomized, open-label, noninferiority trial conducted between 2005 and 2007 at 33 treatment centers in the Netherlands. Patients with permanent AF for up to 12 months' duration and a resting heart rate of over $80 \mathrm{bpm}$ were randomly assigned to a lenient rate-control strategy $(n=311)$, in which the target resting heart rate was below $110 \mathrm{bpm}$, or to strict rate control $(n=303)$ with a target resting heart rate of below $80 \mathrm{bpm}$. Doses of various rate-control drugs (including $\beta$-blockers, calcium-channel blockers, and digoxin) were adjusted until these heart rates were achieved.

Patients were followed up for a maximum of 3 years after enrollment. The target heart rate was achieved for $67 \%$ of patients in the strict-control group and almost all of those in the lenientcontrol group. In this relatively low-risk population (mean $\mathrm{CHADS}_{2}$ score 1.4; $65 \%$ of patients were NYHA class I), there was no significant difference in outcomes, including cardiovascular death, heart failure, and drug-related adverse effects, between the two groups. Neither were there any statistical differences in the number of patients with symptoms, although symptom prevalence did decrease from baseline in both groups.

These results indicate that maintaining a resting heart rate of below $110 \mathrm{bpm}$ may be

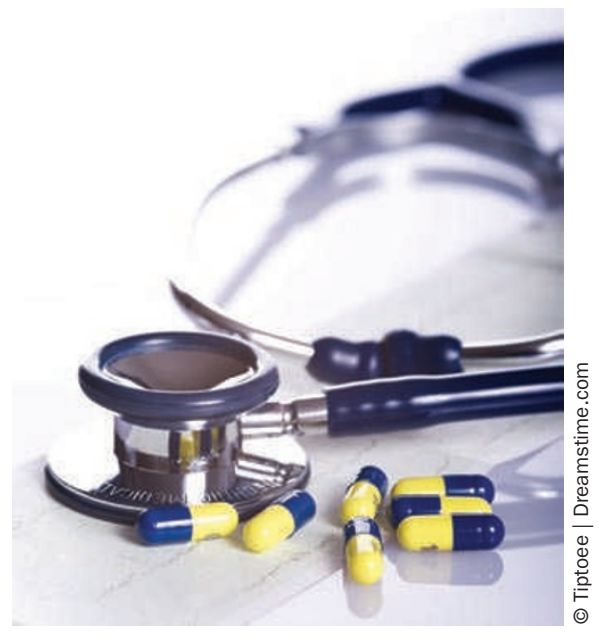

sufficient to prevent cardiovascular events in patients with permanent $\mathrm{AF}$ and, as the investigators note, "lenient control is more convenient, since fewer outpatient visits and examinations are needed."

Alexandra King

Original article Van Gelder, I. C. et al. Lenient versus strict rate control in patients with atrial fibrillation. N. Engl. J. Med. doi:10.1056/NEJMoa1001337 